\title{
Geehrtester Herr Professor!
}

In einem threr Werke: „l'hotographische Resultate einer archäologischen Expedition nach Acgypten" lese ich in den von thnen auf Seite 28 gegebenen Erlïuterungen $z u$ den + Deckenaufnahmen im Pronaos von Dendera folgenden Ausspruch:

„Ieh gehöre keineswegs \%u denjenigen, welehe die so erfreulichen und in der That bedeutenden Fortschritte, die die ägyptisehe lorschung, namentlich in den beiden let\%ten I)ecennien, gemacht hat, gering achten; ich unter- 
schätre gewiss nicht in ihrem Werthe alle die herrlichen Arheiten, welche von Seiten meiner verehrten Herren Fachgenossen auch in Bezug auf die Kalenderfrage bis jetzt erschienen sind; dennoch muss ich, was die dem altägyptischen Kalender und den altägyptisch-astronomischen Denkmälern gewordenen Erklärungen betrifft, bekennen, dass man hier wohl mit grosser Gelehrsamkeit und reeht viel Scharfsinn, aber, wie mir scheinen will, nicht gerade mit besonderem Glück vorwärts gegangen." 
Auch Sie, geehrtester IIerr Professor, sind also der Ansicht, dass, trot\% der bedeutenden Fortschritte auf dem Gebiete der ägyptischen Alterthumsforsehung, man doch in einzelnen Disciplinen noch immer nicht zu sicheren Resultaten habe gelangen können und dass zu diesen Disciplinen auch das altägyptische Kalenderwesen gehöre.

Gestatten Sie, geehrtester Iferr l'rofessor, dass ich, in Anknüpfung an diesen Ihren Ausspruch, als ein Zeichen der Dankbarkeit für die beim Studium Ihrer Werke mir gewordene Anregung und Belehrung, Ihnen meine altägyptischen 
Kalenderstudien widme, in denen ich versuclit habe, den Weg zu zeigen, welchen man bei Reduction igyptischer 7eitangaben einzuschlagen hat.

Genehmigen Sie, geehrtester Herr Professor, den Ausdruck der ausgezeichnetsten Hochachtung von

Ihrem

$$
\text { ergebenen }
$$

$$
\text { August Faselius. }
$$

Weimar, an 2. Februar 1873. 